\title{
Innate Immune Stimulation Should not be Overlooked in Post- exposure Prophylaxis and Early Therapy for Coronavirus Infections
}

\author{
Julio C. Aguilar-Rubido PhD, Eduardo Pentón-Arias MD PhD, Sheikh Mohammad Fazle Akbar MBBS PhD
}

\begin{abstract}
We discuss the suitability of innate immune stimulation in acute respiratory infection post-exposure prophylaxis. The induction of innate immunity can be used to reduce susceptibility to immune-evasive pathogens (coronavirus, influenza virus, respiratory syncytial virus and rhinovirus). After the emergence of multiple SARS-CoV-2 variants, scientists are debating whether new variants could affect vaccine efficacy and how antigens could be redesigned to compensate. In addition, there is insufficient vaccine production to cover universal demand, and equitable vaccine distribution is a global challenge. Given these factors, non-specific immune stimulators may be suitable for a quick first response in the case of a suspected or early respiratory infection. Our group completed several HeberNasvac studies in healthy volunteers and
\end{abstract}

\section{INTRODUCTION}

Innate (or nonspecific) immunity plays a key role in shaping antiviral defenses. RNA viruses are detected by toll-like receptors (TLR), among other innate immune receptors, specifically TLR7 and TLR8, while TLR3 recognizes double-stranded RNA (dsRNA) intermediates formed during viral replication. To avoid detection, coronaviruses (CoVs) and other RNA viruses that cause respiratory infections have developed evasive strategies.[1]

Older adults and people with underlying health conditions are at higher risk of developing severe clinical manifestations of COVID-19. This could be associated with less responsive innate immunity ligands, altered immune responses - both in quality and quantity_changes in the cytokine milieu and lower expression of co-stimulatory signals.[2] Thus, intrinsic host factors may contribute to disease severity and mortality.[3]

Intranasal (IN) stimulation of innate immunity receptors using TLR agonists decreases mortality in mice using the mouse model of lethal infection with SARS-CoV and influenza A virus (IAV).[4,5] Pre- and post-exposure prophylaxes following nonspecific immune stimulation with Hiltonol (a TLR3 agonist), before and after administering a lethal dose of mouse-adapted SARSCoV, resulted in a $100 \%$ survival rate.[4] A second study, using commercial TLR3, TLR7/8 and TLR9 agonists, reduced mortality rates in mouse-adapted SARS-CoV-infected 12-month-old

\footnotetext{
IMPORTANCE

Stimulation of innate immunity against SARS-CoV-2 opens new options for post-exposure prophylaxis and early therapy for other respiratory viruses. This article describes HeberNasvac used as a mucosal innate immunity stimulator and discusses its clinical potential.
}

patients with respiratory infections, and is currently starting large clinical trials in patients with early SARS-CoV-2 infections. This nasal formulation of hepatitis $B$ vaccine has demonstrated its capacity to stimulate innate immunity markers (TLR3, TLR7 and TLR8 in tonsils) at the virus' entry site, in systemic compartments (HLA class II in monocytes and lymphocytes) and in the activation of dendritic cells, lymphocytes and other cell lines in vitro and ex vivo. In addition, research generated by the current pandemic may obtain results useful for treating other acute respiratory infections, which have long been main drivers of mortality among older adults and in early childhood.

KEYWORDS Immunity, innate; toll-like-receptors; SARS-CoV-2; COVID-19; respiratory tract infections; Cuba

mice when they were administered up to 3 days before the lethal SARS-CoV challenge. The TLR3 agonist either completely or partially prevented death in 22-month-old IAV-infected mice following intravenous or intraperitoneal administration, respectively, highlighting the effect of innate immune stimulation even in very old animals.[5] In vitro mouse-adapted SARS-CoV replication inhibition in human epithelial cells treated with a TLR3 agonist, and the reduction in survival of polyinosinic:polycytidylic acid (poly(I:C)-treated TLR3 knockout mice further confirmed the role of TLR3 signaling in the induced protection.[5]

\section{DEVELOPMENT}

HeberNasvac (coded CIGB2020 when used as a non-specific immune stimulator) is a mucosal and parenteral therapeutic vaccine; a liquid formulation containing a recombinant hepatitis $B$ core and surface antigens $\mathrm{HBcAg}$ and $\mathrm{HBsAg}$, produced at the Genetic Engineering and Biotechnology Center (CIGB) in Havana, Cuba. It contains $100 \mu \mathrm{g}$ of each antigen in $1.0 \mathrm{~mL}$ of saline-phosphate buffer. In 2015, Cuba's national regulatory authority approved its use in the country for chronic hepatitis B therapy, and it has also been subject to clinical trials in Japan, Bangladesh and other Asian countries. $[6,7]$ The proteoliposomal and nucleoprotein composition of $\mathrm{HBsAg}$ and $\mathrm{HBcAg}$, respectively, organized in a virus-like structure, facilitates its ability to stimulate dendritic cells[8] and activate B and T cells,[9] which results in a strong adaptive Th1 immune response.[10]

The clinical and pharmacological results of HeberNasvac in hepatitis B patients[6-10] show evidence of innate immune stimulation at the nasopharyngeal and oral mucosa, the main entry sites of SARS-CoV-2. We explored its effect on the local RNA receptor expression (TLR3, TLR7 and TLR8) and other innate immunity markers (Table 1 ).

A phase 1/2 open-label, randomized clinical trial was conducted in 46 hospitalized patients $\geq 60$ years old, of both sexes, with symptoms of respiratory infection $(78.3 \%)$, or asymptomatic, 


\section{\begin{tabular}{|l|l} 
Evidence (Ref. or RPCEC id.) & Study design \\
\hline
\end{tabular} \\ Background: in vitro and in vivo studies}

Immune modulatory and antiviral effects of HeberNasvac[7]

In vitro study of PBMC and pulsed DCs from vaccinated $\mathrm{CHB}$ patients.

Immune modulatory and antiviral effects in vitro and in animal models[8-10]

In vitro stimulation of dendritic cells, B \& T cells.

In vitro studies stimulating HepaRG cells with HBcAg and HeberNasvac.

Multi-agonist effect on TLRs, MyD88/ TRIF pathways and antiviral effects[14]
Objective

Evaluation of cytokines in sera, stimulated PBMC and DCs from CHB patients, as well as the antiviral effect of the product.

Assessing HeberNasvac capacity to increase the markers of innate immunity at molecular and cellular levels and its impact on adaptive immune modulatory response.
Assessment of the capacity of HeberNasvac and their antigens to increase markers of innate immunity at molecular and cellular levels linked to a functional antiviral assay. Treatment: Cells were treated in vitro and in specific cases reinfused to animals.

\section{Results}

Significantly higher levels of IL-1ß; IL-6; IL-8; $\mathrm{IL}-12$ and TNF $\alpha$ than in unvaccinated $\mathrm{CHB}$ patients (control) $(p<0.05)$.

Antiviral effect associated with cytokine stimulation.

Dendritic cells pulsed in vitro induce stronger adaptive immune response \& subvert tolerance when injected in tolerogenic transgenic mice.[8] B \& T cells activation.[9]

Th1 pattern of response after IN administration and Th1 immune modulating effect.[10]

Multi-TLR agonist effect in TLR2, TLR3, TLR7, TLR8, TLR9 cells.

Increased stimulation of adaptors MyD88

TRIF, NF-kb \& IRF3, and molecules involved in antigen presentation (HLA class I, HLA class II, B7.1; B7.2, IFNa and IFNb and other cytokines).These genes increased their expression $(>2 x)$ with statistical signification.

Innate immune stimulation triggered strong antiviral effect in infected HepaRG cells (similar to Entecavir's effects).

Human studies

Safety and local (IN \& SL) effects Open-label, controlled on innate immune markers[11] RPCEC00000306

SARS-CoV-2 pre-exposure prophylaxis: safety and effect[13]

Immune- modulating effect in healthy volunteers[13] and randomized phase $1 / 2$ clinical trial in postexposure prophylaxis of patients with acute respiratory infections or SARS-CoV-2(+).
Open-label, single arm pilot study in healthcare workers and their household contacts.
Assessment of safety and local and systemic immune modulatory effects in a cohort of patients (symptomatic, $78.3 \%$ ) or asymptomatic but exposed to SARS-CoV-2 positive patients. Treatment: 24 patients received HeberNasvac, 3 IN doses on days 0 , 7 and 14 , and a SL dose daily, for 14 days. 22 patients did not receive the product (control group). All patients received the standard treatment.

Safe and well tolerated in patients with acute respiratory infections.

Higher proportion of patients with increased TLR-related genes in tonsils compared to the non-treated controls. Day 8: TLR3, $44.4 \%$ vs. $0 \%$ **; TLR7, $66.6 \%$ vs. $0 \%{ }^{* * *}$ and TLR8, $50.0 \%$ vs. $8.3 \%^{*}$

Higher MFI \% of HLA-class II marker in PBMC's monocytes (55\%; $p=0.023)$ and lymphocytes $(20 \% ; p=0.008)$ in treated vs. non-treated groups.

Preliminary evidence of reduction in number of days with symptoms in treated patients. Mean (SD): 10.12 (9.43) in treated vs. 13.75 (10.80) in controls.

Assessment of the prophylactic effect in 20 highly exposed volunteers by detecting infection or disease worsening in the SARS-CoV-2(+).

Treatment: 20 volunteers received 3 IN doses on days 0,7 , and 14 SL administrations every day.
Open-label, single arm pilot study in volunteers treated with one standard IN administration.
Assessment of the cytokine production of PBMC from 10 healthy volunteers receiving 1 HeberNasvac dose. Treatment: 1 IN administration, extraction after 24 hours.
In a 6 months follow-up period, 4 out of 20 volunteers were SARS-CoV-2(+). The infected patients were older, with comorbidities. Clinical evolution, CT scans, and symptoms evidenced mild progression: 3 patients developed slight cough and 1 patient (55 years old, with diabetes and hypertension) required noninvasive oxygen therapy (2L), symptoms vanished on day 5 . Safety: the treatment was safe and well tolerated, 340 doses, $1.18 \%$ were associated with an $A E$ (nasal drops and sneezing), no systemic $\mathrm{AE}$ reported, all $\mathrm{AE}$ were mild and did not required treatment.

Significant increase in PBMC-induced production of cytokines compared to pre-immune values. IFNg (4.5X); TNFa (9.1X); TGFb (5.4X); IL-2 (2.3X); IL-10 (3.1X).

Early cytokine induction (24 hours after stimulation).

AE: Adverse Events; CHB: chronic hepatitis B; DC: dendritic cells; HBcAg: Hepatitis B core antigen; IN: intranasal; MFI: mean fluorescence intensity;

PBMC: peripheral blood mononuclear cells; RPCEC: Cuban Public Registry of Clinical Trials; SL: sublingual; TLR: Toll-like receptors. The value of $p<0.05\left({ }^{\star}\right) ; 0.01\left({ }^{*}\right)$ and $\left.0.001{ }^{* * *}\right)$ correspond to statistically significant; very significant or highly significant differences. 
but close contacts of SARS-CoV-2 patients.[11] The study was approved by the Center for State Control of Medicines, Equipment and Medical Devices (CECMED) and listed in the Cuban Registry of Clinical Trials, RPCEC00000306-En.[12] Twenty-four patients received HeberNasvac in the study group by both intranasal (IN) and sublingual (SL) routes $(100 \mu \mathrm{g}$ of each antigen in a volume of $1 \mathrm{~mL}$ ). The product was administered daily by SL route for 14 days and by IN route at days 1,7 and 14 . The 22 patients allocated to the control group did not receive HeberNasvac. Both groups received the standard treatment defined by the Cuban Ministry of Health. The adverse events were studied only in vaccinated volunteers. A total of eight different adverse events were detected in five patients $(20.8 \%$ of treated patients). Local adverse events consisted in nasal drops (0.58\%), sneezing $(0.3 \%)$, and otalgia $(0.3 \%)$. Fever $(0.3 \%)$ and asthenia $(0.3 \%)$ were reported as systemic adverse events. None of the adverse events was classified as serious, half of them were unrelated, $25 \%$ possibly related, and $25 \%$ related to the product (nasal drops and sneezing). Most adverse events disappeared without treatment $(62.5 \%)$ or after a pharmacological treatment in the case of fever and otalgia (32.5\%). One patient required treatment due to pneumonia, which was related to the basal condition according to causality analysis. In summary, the administration of HeberNasvac simultaneously by IN and SL routes was safe and well tolerated.

The first PCR test, conducted five days after beginning treatment, detected one positive patient in the treatment group and two in the control group. A subsequent serological study using ELISA detected $50 \%$ participants positive to SARS-CoV-2 in the treated group and $41 \%$ in the control group, suggestive of a previous infection. There was no difference in the evolution of respiratory symptoms. Among the three PCR-positive patients, one from the control group developed pneumonia, diarrhea, edema, and fatigue and after discharge; fatigue and shortage of breath remained for 42 days. The other two patients (one in each group) remained with mild symptoms.[11]

No significant differences appeared in the hematological and blood chemistry analyses between patients treated with HeberNasvac and the control group. Preliminary evidence indicated a reduction in the symptomatic phase in treated patients; a mean of 13.75 (standard deviation, SD 10.80) symptomatic days in the control group vs. 10.12 (SD = 9.43) days in the treated group.[11] IN/SL HeberNasvac administration stimulated innate immune markers at local and systemic compartments; a significant proportion of treated patients had higher gene expression of RNA receptors (TLR3, TLR7 and TLR8) in their oropharyngeal mucosa (tonsils) as well as a significant increase of HLA class II expression in lymphocytes and monocytes from peripheral blood mononuclear cells (PBMC), characterized by $20 \%$ and $55 \%$ increases in mean fluorescence intensity (MFI), respectively[11] (Table 1).

HeberNasvac safety and efficacy in SARS-CoV-2 pre-exposure prophylaxis was studied in two open-label, single-arm pilot studies in a high-incidence area of Dhaka, Bangladesh. The first assessed the drug's prophylactic effect in 20 highly-exposed volunteers (healthcare workers and their household contacts) by detecting infection or disease. Results showed a limited number of infected volunteers after six months' follow-up and mild disease progression in those who tested SARS-CoV-2-positive.[13] The second study was conducted in ten healthy volunteers treated with one standard IN administration of HeberNasvac to determine immunomodulatory and antiviral cytokine expression.

Several immunomodulatory and antiviral cytokines were induced very early, as soon as 24 hours after IN administration[13] (Table 1). A third study in 15 recently-infected SARS-CoV-2 patients immunized as described by Fleites[11] showed no severe COVID-19, and no patient required ventilation.[Aguilar JC, Akbar SMF, clinical trial report, unpublished data] A group of patients with mild or moderate COVID-19 proceeded to severe clinical manifestations of infection during the study. However, these patients remained stable and were discharged from hospital, leading to the approval of a larger clinical trial.

Immunomodulatory effects found in clinical trials were consistent with in vitro immunomodulatory and antiviral effects detected at cellular and molecular levels in previous studies with peripheral blood mononuclear cells (PBMC) and other cells in culture; chronic hepatitis $\mathrm{B}(\mathrm{CHB})$ patients and animal models of immune tolerance[7-9] (Table 1). In particular, HBcAg showed a multitoll-like receptors (multi-TLR) agonist effect, stimulating in vitro dendritic cells and lymphocytes, as well as human hepatoma (HepaRG) cells (infected or not), increasing expression of TLR2, TLR3, TLR7, TLR8 and TLR9 and their signal transduction pathways; MyD88 (Myeloid Differentiation Factor 88), TRIF, IRF3, NF-kB (NF-kappaB).[11,14] The high RNA content associated with $\mathrm{HBcAg}-15 \%$ to $25 \%$ RNA/protein, w/w- stimulates TLR3, TLR7 and TLR8 expression,[11,14,15] implicating stimulation of both MyD88-dependent and TRIF-dependent pathways. This merits the induction of class I/II HLA co-stimulatory molecules B7.1 and B7.2 (CD80/86), type I interferons and other cytokines. Taken together, a set of molecules involved in antiviral immune defense is innervated, consistent with the immunomodulatory and antiviral effects found both in animal models and in vitro.[8-10]

HeberNasvac administration device (dropper vs. spray), posology and dosing are now being optimized in a second study approved by CECMED, the Cuban regulatory authority, for this new use (RPCEC00000326-En, ongoing).[12] Two other protocols have been registered and are pending CECMED approval: a phase $2 / 3$ clinical trial in SARS-CoV-2-positive patients during early stages of infection, aimed at preventing progression to severe COVID-19 (RPCEC00000353-En),[12] and an intervention study on post-exposure prophylaxis for acute respiratory infections (ARI) in older adults (aged $>60$ years) to include persons exposed to SARS-CoV-2, and household contacts of confirmed cases (RPCEC00000356-En).[12] These clinical trials should be completed in 2021-2022.

Recent transcriptomic studies of SARS-CoV-2-in vitro infected cells recommend the use of TLR3 and TLR7/8 agonists for early immune stimulation.[16] Studies in newborn calves infected by bovine coronaviruses have shown the down-regulation of the TLR3 gene, compared to the expression of the same gene in newborn calves infected by bovine rotavirus, further indicating that TLR3 is a target during CoV infection[17] and that stimulation of these TLRs may be appropriate for early COVID-19 therapy and SARS-CoV-2 pre- and post-exposure prophylaxis. The multi-TLR agonistic effect of HBcAg stimulates both MyD88-dependent and independent pathways, resulting in synergistic antiviral responses such as those previously described for the combination of TLR 
Figure 1: Pathways of TLR-mediated activation induced in differentiated HepaRG cells in culture by HBcAg, the antigen of HeberNasvac comprising the RNA ligands of the TLRs involved in RNA-virus recognition (TLR3, TLR7, and TLR8)

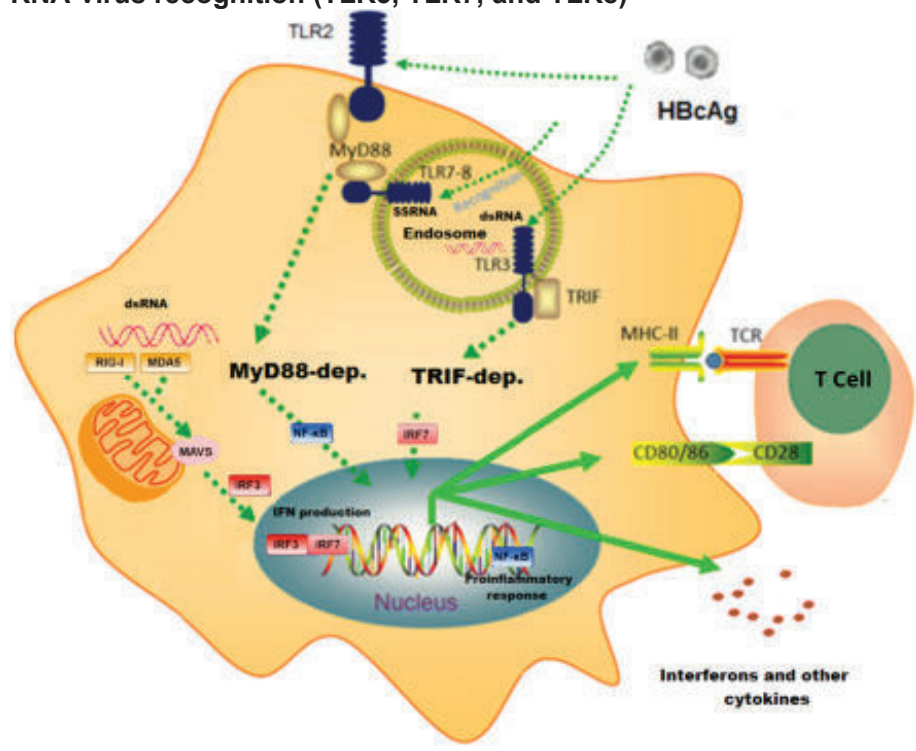

$\mathrm{HBcAg}$ particles, alone or as part of HeberNasvac, stimulate expression of TLRs and other innate immunity markers in cells from oropharyngeal scrapings, as well as several cell types in culture, i.e. dendritic cells, B and T cells and hepatocytes in culture. $[8,9,11,14]$ Signaling through MyD88-dependent and TRIF-dependent pathways results in higher expression of IFN and other proinflammatory cytokines, HLA class I/II stimulation and costimulatory molecules. HBcAg multi-agonist effect is used as a tool to overcome the evasive mechanism described for SARS-CoV-2 and other respiratory pathogens.[1]

ligands.[18,19] The agonistic effect of HBcAg on TLR2 and TLR7 has already been described;[20,21] however, we reported for the first time the simultaneous innervation of TLR3/TLR7 and TLR8 genes, $[11,14]$ a rare quality for a single compound.

HeberNasvac's effect on innate immunity markers was detected as early as day four of treatment and continued up to day eight. Three daily sublingual (on days one to three) and one intranasal (on day one) administrations may be as effective as a schedule of seven administrations for increasing the proportion of patients with higher expression of the receptors and mediators required to detect SARS-CoV-2 and prevent pathogenesis. Such a rapid innervation of the innate immune system is also an attractive property.[13]

Other groups are studying the stimulation of the cytoplasmic RNA receptor RIG-I as a prophylactic and therapeutic protection against SARS-CoV-2 infection in mice.[22] Post-exposure prophylaxis or early therapy in SARS-CoV-2 infections rapidly activate innate immunity, triggering a fast and effective adaptive response, counteracting the virus' evasive mechanisms.[1]

Another SARS-CoV-2 mechanism to evade innate immunity (considered a virus evolutive advantage) was recently described in the variant of concern (VOC) B.1.1.7 (alpha).[23] In this variant, subgenomic RNA overexpresses open reading frames (Orf) proteins Orf9b and Orf6, two well-known innate immunity antagonists,[23] making VOC alpha more effective than the original Wuhan wildtype SARS-CoV-2 variant in suppressing the host's innate immune response in airway epithelial cells. Orf9b and Orf6 interact with TOM70, a mitochondrial protein required for mitochondrial antiviral-signaling protein (MAVS) activation, an RNA-sensing adaptor. The emergence of new VOCs alpha and delta, both of which show increasing transmissibility and severity, further supports the role of immune evasion on pathogenesis, and calls attention to innate immune stimulators for post-exposure prophylaxis and early therapy. HeberNasvac acts to a) improve SARS-CoV-2 RNA detection by stimulating TLR3, TLR7 and TLR8 expression,[11,14] and b) through direct antiviral/immunomodulatory activities as an efficient inducer of interferons and related cytokines.[7-10] TLR-mediated activation is induced by HBcAg, the antigen of HeberNasvac comprising the RNA ligands of the TLRs involved in RNA virus recognition (TLR3, TLR7, and TLR8). Figure 1 describes the pathways of TLR-mediated activation induced in differentiated HepaRG cells by HBcAg, the antigen of HeberNasvac comprising the RNA ligands of the TLRs involved in RNA virus recognition (TLR3, TLR7, and TLR8).

Few products are being evaluated as prophylactic and therapeutic immunomodulators in SARS-CoV-2 infection. The Bacillus Calmette-Guérin (BCG) vaccine was introduced early in the pandemic in several countries as an innate immunostimulant; however, shortages of BCG vaccines and their limited availability for pediatric immunization prevented widespread use.[24] Other immunomodulators being studied are interferons (IFN);[25] their intranasal/inhaled administration has received ongoing attention since the early days of the COVID-19 pandemic. A novel nasal IFN formulation (Nasalferon) received regulatory approval for emergency use in SARS-CoV-2 pre- and post-exposure prophylaxis in Cuba.[26] Since the beginning of the pandemic, two parenterally-administered products, HeberFERON (IFN alfa $2 \mathrm{~b}$ and gamma) and Heberon (IFN alfa $2 \mathrm{~b}$ ), were included in the Cuban guidelines for SARS-CoV-2 treatment.[27]

Local stimulation of the innate immune system by SL administration using preparations of inactivated bacteria has been used to prevent recurrent respiratory infections.[28] IN administration of the adenovirus influenza vaccine prevents death in mice infected with lethal respiratory pathogens.[27] The US Food and Drug Administration (FDA) approved a phase 2 trial of the nasal influenza vaccine candidate T-COVID (Altimmune, Gaithersburg, $M D, U S A)$, a recombinant adenovirus influenza vaccine, for use in early SARS-CoV-2 infection therapy.[29]

In patients with severe COVID-19, HLA-DR expression on monocytes and myeloid dendritic cells (mDCs) is reduced. Plasmacytoid DCs, mDCs and CD14+ monocytes of COVID-19 patients are less responsive to stimulation with bacterial (TLR2/ TLR4/TLR5) and viral (TLR3/TLR7/TLR8) ligand cocktails compared to healthy controls, indicating that innate immune cells in COVID-19 patients are functionally impaired.[30]

HeberNasvac's immunostimulatory effect supports its use in post-exposure prophylaxis and therapy for SARS-CoV-2 and, most likely, for other respiratory infections. Commercial agonists stimulating TLR3, as Poly (I:C) and TLR7/8 (CL097M-012), also innervate antiviral, inflammatory and humoral pathways, decreasing viremia and resulting in clinical improvement in dengue-infected monkeys;[31] in cancer, the relevance of TLR3 stimulation is currently under study.[32] Taken together, the stimulatory properties of HeberNasvac, and HBcAg in particular, may open new prophylactic and therapeutic opportunities. 
A quarter century ago, the molecular mechanisms of the innate immune system were practically unknown. Adjuvants and immunomodulators were selected by trial and error,[33] and some were described as IFN inducers. Today we understand some of these mechanisms, and we know that innate immunity can be trained.[34] Long-term stimulation of innate immune responses (trained immunity), by certain live vaccines, cytokines, toll-like receptors and adjuvants, induces heterologous protection against infection through epigenetic, transcriptional and functional reprogramming of innate immune cells. The induction of trained immunity can be applied to reduce susceptibility to and severity of SARS-CoV-2 infection.[34,35]

Products with HBcAg's demonstrated properties-now initiating phase $2 / 3$ trials-may induce an adaptive immune response if administered soon after infection by viral respiratory pathogens such as SARS-CoV-2. These innate immunostimulatory and modulatory mechanisms are currently used in the development of novel treatments for non-respiratory viruses, as has been the case with HeberNasvac antigens in developing a therapeutic vaccine for HIV, the formulation of which includes HBsAg, $\mathrm{HBcAg}$ and the HIV multiepitopic vaccine candidate CR3;[36] and the development of a nasally-administered vaccine against SARS-CoV-2 named Mambisa that is under study in protocols RPCEC00000345-En and RPCEC00000382-En.[12]

\section{CONCLUSIONS}

Innovative innate immunostimulators such as HeberNasvac may become useful tools for pre- and post-exposure prophylaxis and early therapy in SARS-CoV-2 and other respiratory infections. The knowledge generated by the SARS-CoV-2 pandemic will be useful in confronting respiratory infections in general, which remain a main cause of mortality worldwide. Due to the emergence of SARS-CoV-2 variants, we should be prepared for prolonged coexistence with this pathogen. Innate immunostimulators for nasal or sublingual use, suitable for pharmacy distribution and self-administration, may become a first therapy after symptom onset, and suitable for prophylactic treatment of at-risk contacts, especially among older adults and persons with comorbidities. $-1 /$ -

\section{REFERENCES}

1. Kikkert M. Innate immune evasion by human respiratory RNA viruses. J Innate Immun. 2020 Jan-Feb;12(1):4-20.

2. Metcalf TU, Cubas RA, Ghneim K, Cartwright MJ, Grevenynghe JV, Richner JM, et al. Global analyses revealed age-related alterations in innate immune responses after stimulation of pathogen recognition receptors. Aging Cell. 2015 Jun;14(3):421-32.

3. Neumann-Podczaska A, Al-Saad SR, Karbowski LM, Chojnicki M, Tobis S, Wieczorowska-Tobis K. COVID 19 - Clinical picture in the elderly population: a qualitative systematic review. Aging Dis. 2020 Jul 23;11(4):988-1008.

4. Kumaki Y, Salazar AM, Wandersee MK, Barnard DL. Prophylactic and therapeutic intranasal administration with an immunomodulator, Hiltonol ${ }^{\circledR}$ (Poly IC:LC), in a lethal SARS-CoVinfected BALB/c mouse model. Antiviral Res. 2017 Mar;139:1-12.

5. Zhao J, Wohlford-Lenane C, Zhao J, Fleming E, Lane TE, McCray PB Jr, et al. Intranasal treatment with poly $(1 \cdot \mathrm{C})$ protects aged mice from lethal respiratory virus infections. J Virol. 2012 Nov;86(21):11416-24.

6. Pentón-Arias E, Aguilar-Rubido JC. Cuban prophylactic and therapeutic vaccines for controlling hepatitis B. MEDICC Rev [Internet]. 2021 Jan [cited 2021 Feb 7];23(1):21-9. DOI: 10.37757/MR2021.V23.N1.6. Available at: https://mediccreview.org/estadisticas/ppp.php $? \mathrm{y}=2021 \& \mathrm{v}=1 \& \mathrm{p}=21$

7. Al-Mahtab M, Akbar SM, Aguilar JC, Uddin MH, Khan MS, Rahman S. Therapeutic potential of a combined hepatitis $B$ virus surface and core antigen vaccine in patients with chronic hepatitis $B$. Hepatol Int. 2013 Oct; 7(4):981-9.

8. Akbar SMF, Yoshida O, Chen S, Cesar AJ, Abe $M$, Matsuura $B$, et al. Immune modulator and antiviral potential of dendritic cells pulsed with both hepatitis $B$ surface antigen and core antigen for treating chronic HBV infection. Antivir Ther. 2010;15(6):887-95.

9. Lobaina Y, Hardtke S, Wedemeyer H, Aguilar JC, Schlaphoff V. In vitro stimulation with HBV therapeutic vaccine candidate Nasvac activates $B$ and $T$ cells from chronic hepatitis $B$ patients and healthy donors. Mol Immunol. 2015 Feb;63(2):320-7.

10. Aguilar JC, Lobaina Y, Muzio V, García D, Pentón $\mathrm{E}$, Iglesias $\mathrm{E}$, et al. Development of a nasal vaccine for chronic hepatitis B infection that uses the ability of hepatitis B core antigen to stimulate a strong Th1 response against hepatitis B surface antigen. Immunol Cell Biol. 2004 Oct;82(5):539-46.

11. Fleites YA, Aguiar J, Cinza Z, Bequet-Romero M, Marrero E, Vizcaíno M, et al. HeberNasvac, a therapeutic vaccine for chronic hepatitis $B$, stimulates local and systemic markers of innate immunity: potential use in SARS-CoV-2 post-exposure prophylaxis. Euroasian J Hepatogastroenterol. 2021 Jul-Dec;11(2):59-70.

12. Clinical trials of HeberNasvac and Mambisa indexed at the Cuban Registry of Clinical Trials (RPCEC00000326-En; RPCEC00000353-En; RP CEC00000356-En; RPCEC00000345-En and RPCEC00000382-En) [Internet]. Havana: National Clinical Trials Coordinating Center CENCEC (CU); c2020-2021 [cited 2021 Aug 21]. Available at: https://rpcec.sld.cu/trials/. Spanish.

13. Akbar SMF, Mahtab MA, Aguilar JC, Uddin MH, Khan SI, Yoshida O, et al. Repurposing NASVAC, A Hepatitis B Therapeutic Vaccine, for pre-and post-exposure prophylaxis of SARSCoV-2 infection. J Antivir Antiretrovir. 2021 May;13(S20):1000004.

14. Aguilar JC, Aguiar J, inventors; Center for Genetic Engineering and Biotechnology, assignee. Viral nucleoprotein and formulations thereof. Cuba patent application CU 2020-0028. 2020 April 20.

15. Aguilar JC. A therapeutic perspective of the immunological function of the liver. Biotecnol Apl. 2010 Jan;27(1):10-8.

16. Prasad K, Khatoon F, Rashid S, Ali N, A Asmari $A F$, Ahmed $M Z$, et al. Targeting hub genes and pathways of innate immune response in COVID-19: a network biology perspective. Int J Biol Macromol. 2020 Nov 15;163:1-8.

17. Aich P, Wilson HL, Kaushik RS, Potter AA, Babiuk LA, Griebel P. Comparative analysis of innate immune responses following infection of newborn calves with bovine rotavirus and bovine coronavirus. J Gen Virol. 2007 Oct;88(Pt 10):2749-61.

18. Zhu Q, Egelston C, Gagnon S, Sui Y, Belyakov IM, Klinman DM, et al. Using 3 TLR ligands as a combination adjuvant induces qualitative changes in $T$ cell responses needed for antiviral protection in mice. J Clin Invest. 2010 Feb;120(2):607-16.

19. Zhu Q, Egelston C, Vivekanandhan A, Uematsu S, Akira S, Klinman DM, et al. Toll-like receptor ligands synergize through distinct dendritic cell pathways to induce $\mathrm{T}$ cell responses: implications for vaccines. Proc Natl Acad Sci U S A. 2008 Oct; 105(42):16260-5.

20. Cooper A, Tal G, Lider O, Shaul Y. Cytokine induction by the hepatitis $B$ virus capsid in macrophages is facilitated by membrane heparan sulfate and involves TLR2. J Immunol. 2005 Sep;175(5):3165-76.

21. Lee BO, Tucker A, Frelin L, Sallberg M, Jones $J$, Peters C, et al. Interaction of the hepatitis B core antigen and the innate immune system. J Immunol. 2009 Jun;182(11):6670-81.

22. Mao T, Israelow B, Lucas C, Vogels CBF, Fedorova O, Breban MI, et al. A stem-loop RNA RIG-I agonist confers prophylactic and therapeutic protection against acute and chronic SARS-CoV-2 infection in mice. bioRxiv 448754 [Preprint]. 2021 Jun 17 [cited 2021 Jun 26]. Available at: https:// doi.org/10.1101/2021.06.16.448754

23. Thorne LG, Bouhaddou M, Reuschl AK, ZulianiAlvarez L, Polacco B, Pelin A, et al. Evolution of enhanced innate immune evasion by the SARSCoV-2 B.1.1.7 UK variant. bioRxiv 446826 [Preprint]. 2021 Jun 7 [cited 2021 Jun 16]. Available at: https://doi.org/10.1101/2021.06.06.446826.

24. Curtis N, Sparrow A, Ghebreyesus TA, Netea MG. Considering BCG vaccination to reduce the impact of COVID-19. Lancet. 2020 May;395(10236):1545-6.

25. Iturriaga C, Eiffler N, Aniba R, Ben-Othman R, Perez-Mateluna G, Meyer JKV, et al. A cluster randomized trial of interferon $ß-1 \mathrm{a}$ for the reduction of transmission of SARS-Cov-2: protocol for the Containing Coronavirus Disease 19 trial (ConCorD-19). BMC Infect Dis. 2021 Aug 13;21(1):814.

26. Nasalferon [Recombinant Human Interferon Alpha 2b]. Summary of Product Features. [Internet]. Havana: Center for State Control of Medicines, Equipment and Medical Devices CECMED (CU) ; c2021 [cited 2021 Aug 21]. Available at: https://www.cecmed.cu/registro/rcp/ nasalferon-Interferon-alfa-2b-humano-recombi nante. Spanish.

27. Pereda R, González D, Blas Rivero H, Rivero JC, Pérez A, Del Rosario López L, et al. Therapeutic effectiveness of interferon- $\alpha 2 b$ against COVID-19: the Cuban experience. J Interferon Cytokine Res. 2020 Sep;40(9):438-42. 
28. Sánchez Ramón S, Manzanares M, Candelas G. MUCOSAL anti-infections vaccines: beyond conventional vaccines. Reumatol Clin (Engl Ed). 2020 Jan-Feb;16(1):49-55. English, Spanish.

29. Altimmune Receives Award from U.S. Department of Defense to Fund Phase 1/2 Clinical Trial of T-COVID ${ }^{\mathrm{TM}}$ in Outpatients with Early COVID-19 [Internet]. Gaithersburg: Altimmune Headquarters; 2020 Jun 29 [cited 2021 Jun 29]; [about 2 p.]. Available at: https://www.globenewswire com/news-release/2020/06/29/2054563/0/en/ Altimmune-Receives-Award-from-U-S-Depart ment-of-Defense-to-Fund-Phase-1-2-Clinical -Trial-of-T-COVID-in-Outpatients-with-Early -COVID-19.htm

30. Arunachalam PS, Wimmers F, Mok CKP, Perera RAPM, Scott M, Hagan T, et al. Systems biological assessment of immunity to mild versus severe COVID-19 infection in humans. Science. 2020 Sep 4;369(6508):1210-20.

31. Sariol CA, Martínez MI, Rivera F, Rodríguez IV, Pantoja P, Abel K, et al. Decreased dengue replication and an increased anti-viral humoral response with the use of combined Toll-like receptor 3 and $7 / 8$ agonists in macaques. PLoS One. 2011 Apr 29;6(4):e19323.

32. Yuan MM, Xu YY, Chen L, Li XY, Qin J, Shen Y TLR3 expression correlates with apoptosis, proliferation and angiogenesis in hepatocellular carcinoma and predicts prognosis. BMC Cancer. 2015 Apr 9;15:245. DOI: 10.1186/s12885-015-1262-5

33. Aguilar JC, Rodríguez EG. Vaccine adjuvants revisited. Vaccine. 2007 May 10;25(19):3752-62.
34. Sui Y, Berzofsky JA. Myeloid cell-mediated trained innate immunity in mucosal AIDS vaccine development. Front Immunol. 2020 Feb 28:11:315. DOI: 10.3389/fimmu.2020.00315

35. Netea MG, Giamarellos-Bourboulis EJ, Domínguez-Andrés J, Curtis $\mathrm{N}$, van Crevel $\mathrm{R}$, van de Veerdonk FL, et al. Trained immunity: a too for reducing susceptibility to and the severity of SARS-CoV-2 infection. Cell. 2020 May 28;181(5):969-77.

36. Iglesias E, Thompson R, Carrazana Y, Lobaina $Y$ García D, Sánchez J, et al. Coinoculation with hepatitis B surface and core antigen promotes a Th1 immune response to a multiepitopic protein of HIV-1. Immunol Cell Biol. 2006 Apr;84(2):174-83.

\section{THE AUTHORS}

Julio César Aguilar-Rubido (Corresponding author: julio.aguilar@cigb.edu.cu), biochemist with a doctorate in biological sciences. Senior researcher and head of the Hepatitis B Group, Vaccine Department, Biomedical Research Division, Genetic Engineering and Biotechnology Center (CIGB), Havana, Cuba. https://orcid.org/0000-0003-0166-4784

Eduardo Pentón-Arias, physician specialized in clinical biochemistry with a doctorate in biological sciences. Senior researcher, Latin American School of Medicine (ELAM) and
Hepatitis B Group, Vaccine Department, Biomedical Research Division, CIGB, Havana, Cuba. https://orcid.org/0000-0002-0574-7942

Sheikh Mohammad Fazle Akbar, physician specializing in immunology with a doctorate in biological sciences. Senior researcher, Department of Gastroenterology and Metabology, Ehime University Graduate School of Medicine, Ehime, Japan 791-0295. https:// orcid.org/0000-0002-8139-2645

Submitted: April 13, 2021

Approved for publication: December 6, 2021 Disclosures: Julio César Aguilar-Rubido and Eduardo Pentón-Arias are employed at the Genetic Engineering and Biotechnology Center (CIGB). CIGB developed and produces HeberNasvac (CIGB2020), HeberFERON, Nasalferon and Heberon. 